\title{
Experiment synthesis and characterization of acoustic instabilities in high intensity discharge
}

\begin{abstract}
High intensity discharge lamps comprise a gas encapsulated in a transparent vessel, in which a light emitting discharge arc is established. The lamp operation necessitates a driver that provides an energy saving and cost reduction potential when the frequency of the alternating current is tuned from the commonly used low frequency range to a higher frequency range. However, such an operation can result in low frequency luminous fluctuations, lamp extinction or even destruction. The excitation of acoustic resonances in the high frequency range is the reason for the instable behaviour; therefore a predictive model was established in order to give us at which frequencies light flicker can be expected. Moreover, we conducted an experimental study, which allows the electrical detection of frequency regions, in which the discharge arc behaves instable. Furthermore, an optical system is incorporated to record images of the discharge arc during stable and instable operating conditions. The results enable a considerably better understanding of the flicker phenomenon in HID lamps and facilitate the development of energy efficient drivers.
\end{abstract}

Keywords: arc tube, HID lamp, frequencies light flicker, electric potential drop, acoustic resonance, arc motion, pressure waves.
Volume 7 Issue I - 202 I

\author{
AMER L,' HAMOUDA M,' Jöerg Shwieger² \\ 'Department of Material Sciences, University of Adrar, Algeria \\ ${ }^{2}$ Department of Mechanical Engineering and Production, \\ Heinrich Blasius Institute for physical, Germany
}

Correspondence: AMER L, Department of Material sciences, University of Adrar, Algeria, Tel 213663763440 ,

Email lakhdarame@yahoo.fr

Received: December 22, 2020 | Published: Febrauary 25, 202

\section{Introduction}

High-intensity discharge lamps include high-pressure sodium and high pressure mercury lamps. These light sources emit visible radiation by energy exchanges of excited atomic states in a discharge arc. HID lamps are characterised by a static pressure inside the lamp that exceeds the ambient pressure during steady state operation. The main constituent of the discharge arc distinguishes the sodium from the mercury lamp. Additionally, these lamps usually contain metal halides to improve the light quality. Metal halide lamps are further divided in to quartz metal halide and ceramic metal halide lamps dependent on the tube material that envelops the discharge arc. The sun like luminance, the compact size and the high colour quality of HID lamps will guarantee a market for these lamps in the future. Moreover, the supply of replacement lamps for existing lighting systems will maintain a worthwhile market. In the product life cycle concept, HID lamp systems evolve to the mature stage. This means that cost becomes the key focus of many development activities and that drivers for operating HID lamps are optimised to cheaper designs. Minimal driver costs and an increased efficiency can be achieved by operating the lamp at an $\mathrm{AC}$ with a frequency of approximately $300 \mathrm{kHz}$, but in this frequency range acoustic resonances, that lead to arc flickering, are particularly distinctive. Movements of the discharge arc are caused by the excitation of standing pressure waves inside the arc tube that result from periodic heating when operated at an alternating current. Therefore, these discharge instabilities are also called acoustic instabilities. If the discharge arc is acoustically excited, depends on the pressure distribution of the acoustic eigenmode so that arc movements can not be detected at every acoustic eigenfrequency. A predictive model was established, translated into calculation program under the Matlab language through which we could determine the oscillations frequencies for the fundamental propagation modes. For a thorough understanding of the instabilities, accompanying experimental investigations have to be conducted. These investigations should be able to electrically and optically quantify the discharge arc at stable and instable operating conditions. Especially, changes during acoustic excitation of the arc tube content should be detected. The results of the experiments and simulations have to be discussed.

\section{Physical modelling of acoustic resonance}

The shape of the discharge arc in the HID lamp depends on many factors: Arc tube geometry, electrode distance, absolute pressure inside the arc tube, type and amount of metal halides, mounting position, input power, etc. These factors influence the arc constriction, the arc length, the temperature distribution, the acoustic eigenmodes and their corresponding frequencies as well as many other physical fields. To increase the light quality and the conversion efficiency from electric power to visible light, the lamp can be operated at higher halogen pressures. However, these results in constricted arcs that is more susceptible to acoustic instabilities. The proposed physical model of acoustic resonance, allows predicting excitation conditions of acoustic resonance and the arc form. This model is obtained when considering the discharge in the lamp as plasma in Local Thermodynamic equilibrium (LTE). Under these conditions all the plasma's sizes are a temperature function often very complicated. A precise measurement carried out within the laboratory to obtain the geometrical profile of the plasma temperature is necessary for the determination of the acoustic resonance frequencies. We can completely model the behaviour of the discharge by using the conservation relations of the mass, momentum and energy, coupled with electric relations and those of the radiation. Considering the loss by friction due to the plasma viscosity insignificant, which means that we can omit the terms of amortization, which leads to the following equation, characterizing the propagation of the pressure waves in the plasma.

$$
\begin{gathered}
\frac{\partial^{2} p}{\partial t^{2}}-C_{s}^{2} \Delta p=(\gamma-1) \frac{\partial N}{\partial t} \\
N=P_{\text {ele }}-U_{\text {ray }}-W_{\text {th }}
\end{gathered}
$$




$$
C_{s}=\sqrt{\gamma \cdot \frac{{ }^{R} M \cdot T}{M}}
$$

The equation (1) is very complex and requires the knowledge of a great number of data and its solution is extremely difficult. However, if our reasoning is limited just to the prediction of frequencies where the acoustic resonances appear, we then can omit the term source which depends only on the plasma. So we will treat the propagation of pressure wave in a hot gas but not ionized. In this context certain terms of the model may be neglected and the equation is considerably simplified. After simplification, we gets:

$$
\nabla^{2} p=\frac{1}{C_{s}^{2}(T)} \cdot \frac{\partial^{2} p}{\partial t^{2}}
$$

This simplified formulation, known as "Helmholtz equation" makes it possible to determine the acoustic resonance frequencies. If, initially we consider that the temperature and the speed of propagation of sound are constant. Under these conditions this equation can be analytically solved, in a cylinder of ray $\mathrm{R}$ and length $\mathrm{L}$, by the variables separation method:

$$
\begin{aligned}
P(r, \phi, z, t) & =P_{A} J_{n}\left(\frac{W_{r} r}{C_{s}}\right) \cos (n \phi) \cos \left(\frac{W_{z} Z}{C_{s}}\right) e^{-j \omega t} \\
\omega_{n l m} & =\sqrt{\left(\frac{a_{n m} C_{s}}{R}\right)^{2}+\left(\frac{\pi l C_{s}}{L}\right)^{2}}
\end{aligned}
$$

According to the equation (6), the acoustic resonance frequency depends then on the dimensions of the discharge tube (ray R and length $\mathrm{L}$ ), and the celerity of the pressure propagation Cs which itself depends on the composition of gases and average temperature of the plasma. This means that the resonance frequency may vary with the ageing of the lamp because of the change of gas compositions, and with the temperature which represents the total power injected into the discharge. Consequently, because of the manufacture tolerance, we can have light differences in acoustic resonance frequencies for lamps of the same type and manufacturer. For the equation (5), terms (n,m,l) represent as well the spatial distribution of pressure in the discharge, by indicating $\omega_{m}=\omega_{r}$ the transverse frequency of resonance according to $(r, \varphi)$, by $\omega_{l}=\omega_{z}$ the longitudinal frequency of resonance according $\mathrm{z}$, and by $\omega_{n, m, l}=\omega$ the combined resonance frequency or global. The equation (5) enables us to distinguish the following terms:

$$
P(r, \phi, z, t)=P_{A} P_{n}^{\text {Amplitude }}\left[J_{n}\left(\frac{W_{r} r}{C_{s}}\right)\right][\cos (n \phi)]\left[\cos \left(\frac{W_{z} Z}{C_{S}}\right)\right]\left[e ^ { \text { terme Azimuthal } } \left[\begin{array}{c}
\text { terme Longitudinal } \\
\text { Propagation }
\end{array}\right.\right.
$$

\section{Experimental setup}

The experimental setup was mainly used to determine the acoustic eigenfrequencies of the arc tube of the HID lamp. Additionally, the setup served to investigate the hysteresis effect. Optical measurement devices were implemented in this setup to detect the emitted light of the discharge arc during stable and unstable operation. Figure 1. schematically depicts the experimental setup that consists of the HID lamp itself, the equipment necessary to operate the lamp and different measurement devices. Furthermore, the manufacturer and the product name are presented. The HID lamp, a Philips MASTER Colour CDM-T Elite 35W/930, was operated at a square-wave voltage with a carrier frequency $\mathrm{fc}=400 \mathrm{~Hz}$. The square wave signal was provided by an Agilent 33220.A function generator with rise and fall time of less than $13 \mathrm{~ns}$ and an over shoot of less than $2 \%$. No instabilities of the discharge arc occurred at fc because the driving frequency was considerably smaller than the lowest acoustic eigenfrequency at approximately $42 \mathrm{kHz}$. The second function generator, a Wavetek Mode 29A, produced a sinusoidal voltage with a resolution of $0.1 \mathrm{mHz}$ that was used to excite discharge arc flicker. For this reason, the frequency of this signal is also called excitation Frequency $f_{\text {ex. }}$

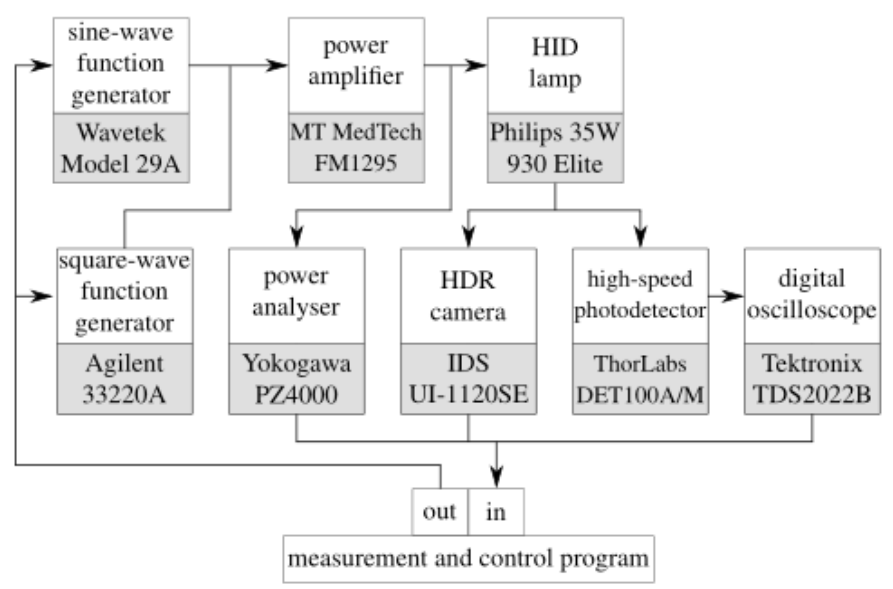

Figure I Experimental setup to characterise discharge arc flicker in HID lamps.

It was tuned to different frequencies in a certain high frequency range. The power amplifier, a MTMedTech FM 1295, regulated the amplitude of the voltage $V(t)$ and the electric current $I(t)$ so that the HID lamp was operated at its nominal power. The modulation depth $\alpha$ describes the ratio of the sinusoidal to the square wave voltage amplitude. The power can be derived from $\mathrm{P}(\mathrm{t})=\mathrm{V}^{2}(\mathrm{t}) / \mathrm{R}_{\text {plasma }}$ with the electric resistance of the plasma $\mathrm{R}_{\text {plasma }}$. The power analyser, a Yokogawa PZ 4000, measured the following data : The electric current, the electric potential drop between the electrodes and the electric power. The high dynamic range (HDR) camera, a IDSUI-1120SE, enables direct observation of the discharge arc because it can resolve the high brightness differences between the arc and its surrounding. The CMOS chip of the camera is sensitive in the wave length range of $400 \mathrm{~nm}$ to $900 \mathrm{~nm}$ with the highest sensitivity at $710 \mathrm{~nm}$, has a resolution of $768 \times 576$ pixels and a high dynamic range of $120 \mathrm{~dB}$. The maximal refresh rate of the camera is $50 \mathrm{~Hz}$. When the discharge arc flicker was investigated experimentally, both the camera and the high speed silicon photodetector, a DET100A/M from ThorLabs, were used. The photodiode with a rise time of $43 \mathrm{~ns}$ converts the brightness fluctuations into an electric current. The spectral sensitivity is $400 \mathrm{~nm}$ to $1100 \mathrm{~nm}$ with a peak wave length at $970 \mathrm{~nm}$. The photodiode was placed in a distance of $0.2 \mathrm{~m}$ from the HID lamp to receive enough illumination and simultaneously to prevent saturation. The digital oscilloscope, a Tektronix TDS2022B, records the time dependent signal and converts it into the frequency domain by fast Fourier transform (FFT). A computer transmitted the values of the modulation depth and the excitation frequency to the function generators and records the camera and photodiode signals as well as the electric 
current, electric potential drop and electric power. To control the experimental setup, program code created with MATLAB was used. To determine the acoustic eigenfrequencies that lead to a flickering discharge arc, the lamp was initially operated at a modulation depth of $0 \%$ for at least ten minutes. The lamp reached a stationary state at this stable condition, which means that the temperature inside the arc tube, the voltage drop between the electrodes, their radiated light, do not change anymore. The constant electric potential drop between the electrodes without acoustic excitation is defined as the reference voltage $\mathrm{V}_{\text {ref* }}$

In case of the exemplary measurement in Figure 2, $\mathrm{V}_{\text {ref }}$ is $90 \mathrm{~V}$ $\left(f_{e x}=40.0 \mathrm{kHz}, \alpha=0 \%\right)$. The electric potential is proportional to the arc length because the passage of current through the plasma acts as an ohmic resistance. Therefore, arc flicker can directly be observed by measurement of the voltage drop. After the stationary state was reached, $\mathrm{f}_{\text {ex }}$ was set to a constant value near an acoustic eigenfrequency of the lamp and $\alpha$ was step wise increased every $10 \mathrm{~s}$ to excite acoustic waves. Meanwhile, the potential drop was measured every $0.5 \mathrm{~s}$, and the power was regulated to keep it at its nominal value. When the voltage fluctuated more than $\mathrm{V}_{\text {fluc }}=1.5 \mathrm{~V}$ or when the voltage exceeded $\mathrm{V}_{\lim }=\mathrm{V}_{\text {ref }}+5.0 \mathrm{~V}$, the measurement was aborted to prevent lamp failure caused by exceedingly high arc tube temperature or by temperature oscillations of the arc tube. Immediately afterwards, $\alpha$ was set back to $0 \%$ and $\mathrm{f}_{\mathrm{ex}}$ was increased to the next frequency step. Figure 2 . displays both cases of measurement abortion. A voltage fluctuation of more than $\mathrm{V}_{\text {fluc }}$ occurs at $\mathrm{f}_{\text {ex }}=40.0 \mathrm{kHz}$ and $\alpha=6 \%$, and the voltage limit $\mathrm{V}_{\text {lim }}$ is exceeded at $\mathrm{f}_{\text {ex }}=40.5 \mathrm{kHz}$ and $\alpha=10 \%$. For the $35 \mathrm{~W}$ lamp, the excitation frequency was generally increased from $35 \mathrm{kHz}$ to 50 $\mathrm{kHz}$ in $500 \mathrm{~Hz}$ steps to excite the first instability at around $42 \mathrm{kHz}$. The modulation depth was increased from $0 \%$ to $12 \%$ in $2 \%$ steps.

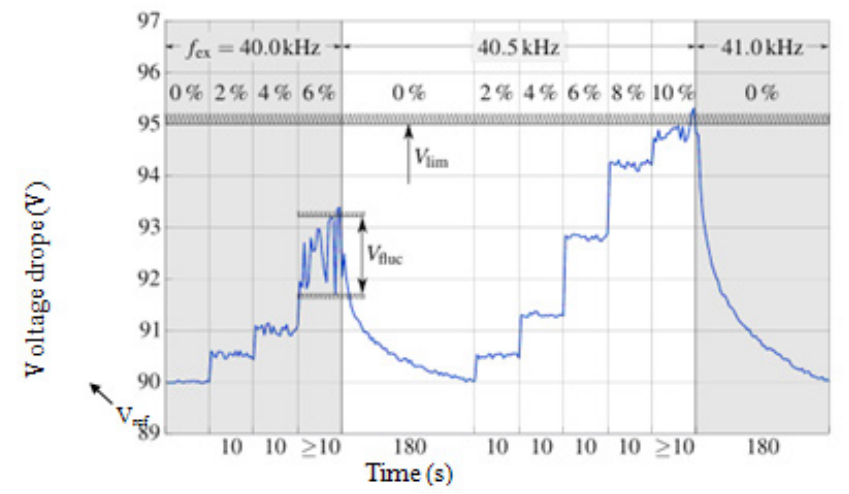

Figure 2 Exemplary behaviour of the voltage drop during determination of acoustic eigenfrequencies.

\section{Simulation results}

Table 1.

Table I Lamps characteristics

\begin{tabular}{ll}
\hline $\begin{array}{l}\text { Standard lamp SHP } \\
\text { 400W }\end{array}$ & $\begin{array}{l}\text { standard lamp VMHP 400W } \\
\text { with 70mg }\end{array}$ \\
\hline$R=3,75 \mathrm{e}^{-3} \mathrm{~m}$. & $R=9,5 \mathrm{e}^{-3} \mathrm{~m}$ \\
$\mathrm{~L}=10,7 \mathrm{e}^{-2} \mathrm{~m}$. & $\mathrm{L}=8,2 \mathrm{e}^{-2} \mathrm{~m}$ \\
$C_{\mathrm{s}}=470,7 \mathrm{~m} / \mathrm{s}^{2}$ & $C_{s}=491,71 \mathrm{~m} / \mathrm{s}^{2}$ \\
\hline
\end{tabular}

\section{SHP lamp}

Figure 3- Figure 5.

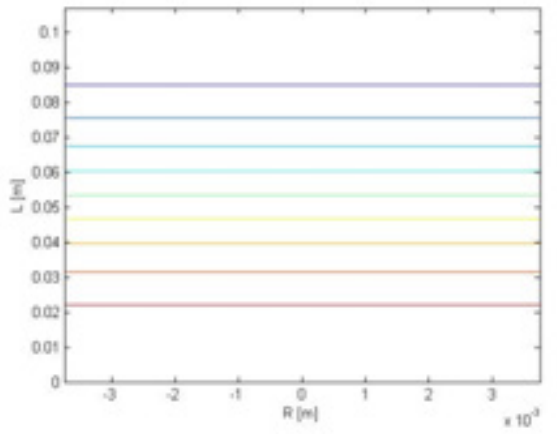

Figure 3 Longitudinal fundamental mode $(0,0,1)$.

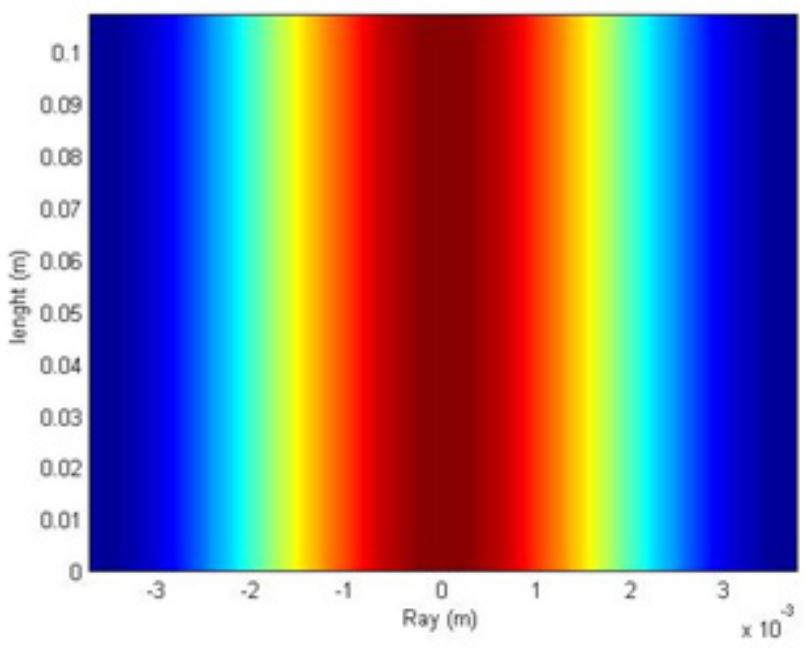

Figure 4 Radial fundamental mode $(0,1,0)$

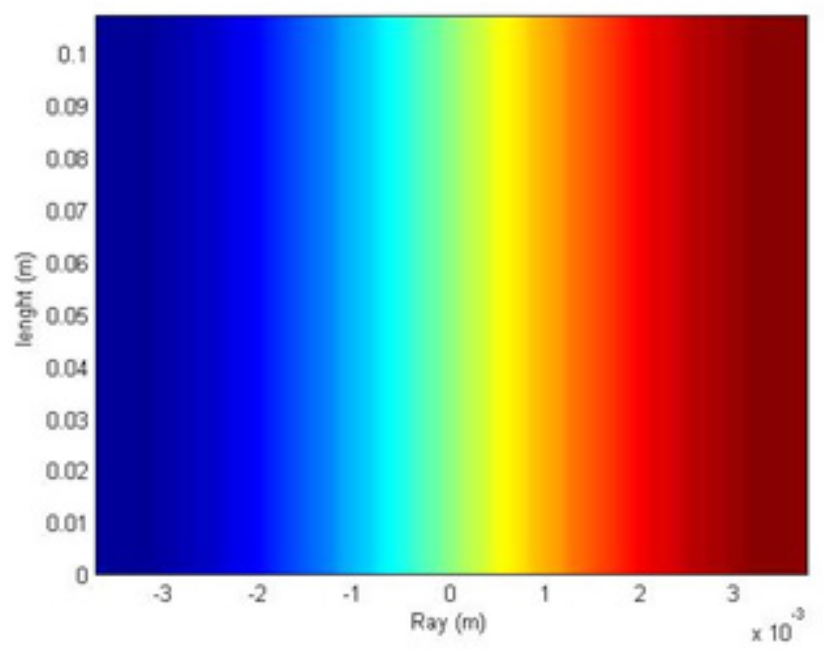

Figure 5 Azimuth fundamental mode $(1,0,0)$.

\section{VMHP results}

Figure 6- Figure $8 \&$ Table 2. According to the distribution 
of pressure in the arc and we note to the theory that the discharge endeavours to move through zones where the pressure is low. The arc takes the way which corresponds to the least losses. For longitudinal mode $\mathrm{Z}$ axial $(0,0,1)$ we can see clearly that the pressure begins to take important values starting from $0.045 \mathrm{~m}$ for the HPS lamp whereas for the HPMV lamp it starts from $0.035 \mathrm{~m}$. For radial longitudinal mode $\mathrm{R}$ axial $(0,1,0)$, the higher pressures lies between $-0,8 \cdot 10^{-3} \mathrm{~m}$ and $0,8 \cdot 10^{-3} \mathrm{~m}$ for HPS lamps type and between $-2 \cdot 10^{-3} \mathrm{~m}$ and $2 \cdot 10^{-3} \mathrm{~m}$ for HPMV lamps type. The $\varphi$ axial, azimuth mode $(1,0,0)$ the pressure starts to get important values from $2,5.10^{-3} \mathrm{~m}$ for HPS lamp while for the HPMV this value is equal to $5,8 \cdot 10^{-3} \mathrm{~m}$.Concerning the two modes longitudinal Z-axial $(0,0,1)$ and R-radial $(0,1,0)$, we remark well that the probability to get an acoustic resonance effect is important in the case of HPMV lamps type than that of HPS ones, then it is reversed for the $\varphi$ axial azimuth mode $(1,0,0)$. In experiments this effect is translated as follow, the Acoustic resonance in longitudinal mode arises by a curved arc at the level of one of the discharge ends. In radial mode, the arc seems to segment successively to diffuse zones then narrow. Finally, the azimuth mode is an oscillation of the arc from one end to another.

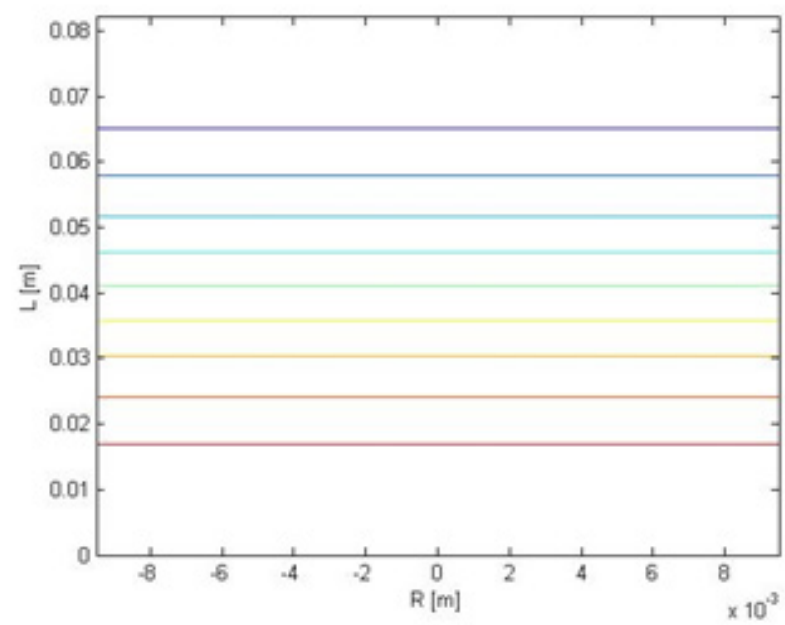

Figure 6 Longitudinal fundamental mode $(0,0, I)$.

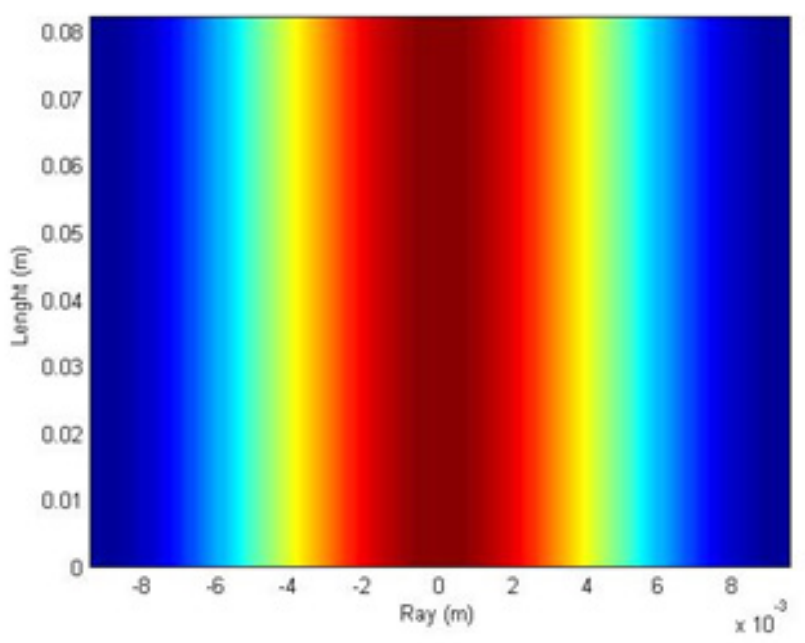

Figure 7 Radial fundamental mode $(0,1,0)$.

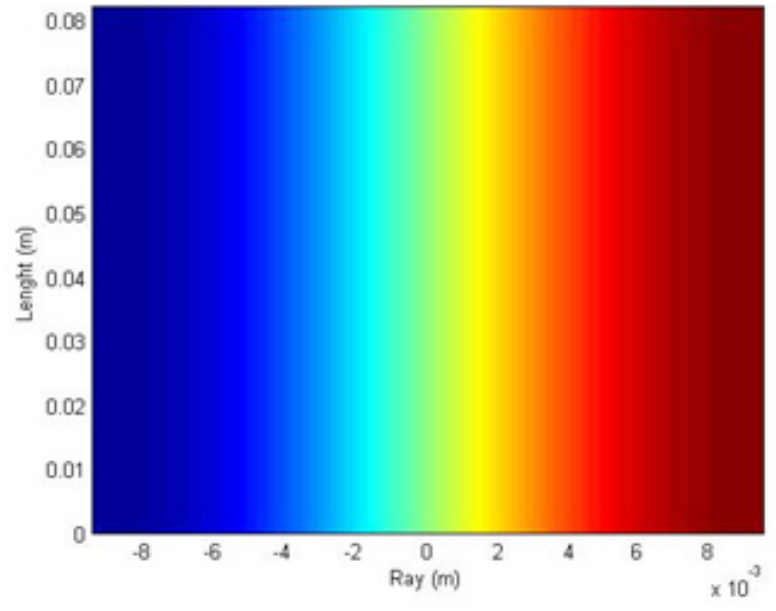

Figure 8 Azimuth fundamental mode $(I, 0,0)$.

For all graphs: $\square$ high pressure

$\square$ Null pressure

Low pressure

Table 2 Frequencies of the acoustic resonances

\begin{tabular}{|c|c|c|c|}
\hline $\begin{array}{l}\text { Mode } \\
\text { Lamp type }\end{array}$ & $(0,0,1)$ & $(0,1,0)$ & $(1,0,0)$ \\
\hline $\begin{array}{l}\text { SHP } \\
400 W\end{array}$ & $2.1995 \mathrm{kHz}$ & $76.5464 \mathrm{kHz}$ & $\begin{array}{l}36.7819 \\
\mathrm{kHz}\end{array}$ \\
\hline $\begin{array}{l}\text { VMHP } \\
400 W\end{array}$ & $2.9982 \mathrm{kHz}$ & $31.5644 \mathrm{kHz}$ & $\begin{array}{l}15.1672 \\
\mathrm{kHz}\end{array}$ \\
\hline
\end{tabular}

\section{Experimental results}

Figure $9 \&$ Figure 10. Exemplary light intensity measurement of a horizontally operated HID lamp. The black line indicates the mean position of all light intensity values that are larger than $95 \%$ times the highest light intensity as a function of the y coordinate. The main results of the optical measurements are presented in Figure.10 The fluid flow bends the discharge arc upwards so that an arc deflection of $0.99 \mathrm{~mm}$ at $\mathrm{y}=0 \mathrm{~mm}$ occurs in the exemplary image shown on the figure. Additionally, this image illustrates the translucency of the tube wall: The image is not sharp, but blurred. As the arc tube consists of two welded half-shells, the weld seam refracts the light. Consequently, a lower intensity in the z-direction is measured that appears as a vertical stripe at $\mathrm{y} \approx 0 \mathrm{~mm}$. Figure 11 presents an overview of a wide frequency range of $20 \mathrm{kHz}$ to $200 \mathrm{kHz}$, in which arc flicker in the investigated HID lamp occurs. The modulation depth in this experiment was varied from $0 \%$ to $10 \%$ in $1 \%$ steps. In the grey coloured area, no arc flicker was detected and, hence, the lamp could stably be operated. The maximum modulation depth was not reached at some excitation frequencies because the voltage drop exceeds one or even both termination criteria. These consist of the voltage fluctuation $\mathrm{V}_{\text {fluc }} \geq 1.5 \mathrm{~V}$ measured at one operating point (specific excitation frequency fex and modulation depth $\alpha$ ) and of a voltage limit of $\mathrm{V}_{\text {lim }} \geq \mathrm{V}_{\text {ref }}+5.0 \mathrm{~V}$ that exceeds the reference voltage measured at a modulation depth of $0 \%$. Thus, the termination prevents operation 
at higher modulation depths at this excitation frequency to prevent lamp failures that are caused by pressure fluctuations or exceedingly high pressures inside the arc tube. With regard to the amplitude (modulation depth), the experimental results show an increasing loss with an increasing excitation frequency because the modulation depth necessary to excite flicker increases. We notice that up to $120 \mathrm{kHz}$ a modulation depth of $4 \%$ is sufficient to excite flicker, but a higher modulation depth is necessary at frequencies beyond $120 \mathrm{kHz}$ (Figure 12).

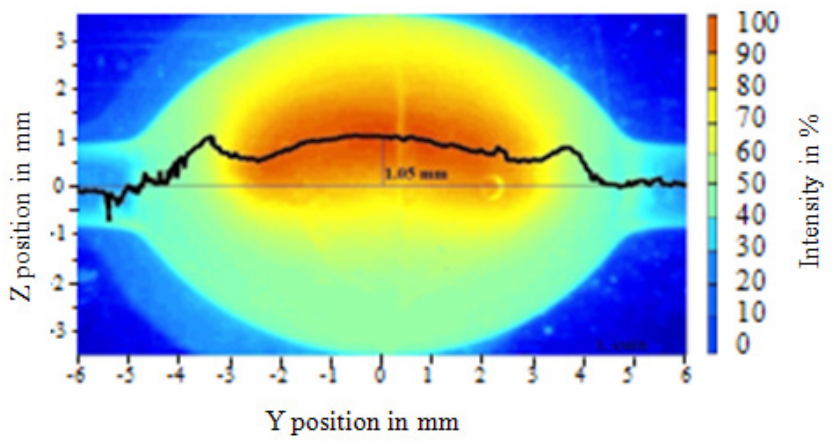

Figure 9 Experimental determination of arc deflection at unstable operating conditions.

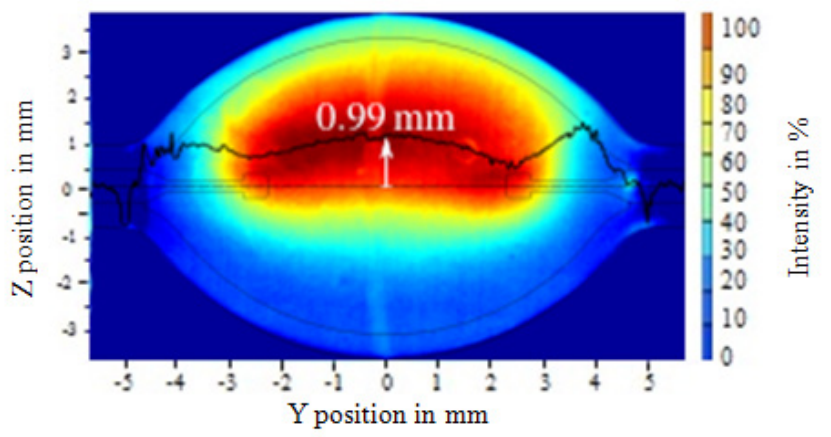

Figure 10 Experimental determination of arc deflection at stable operating conditions.

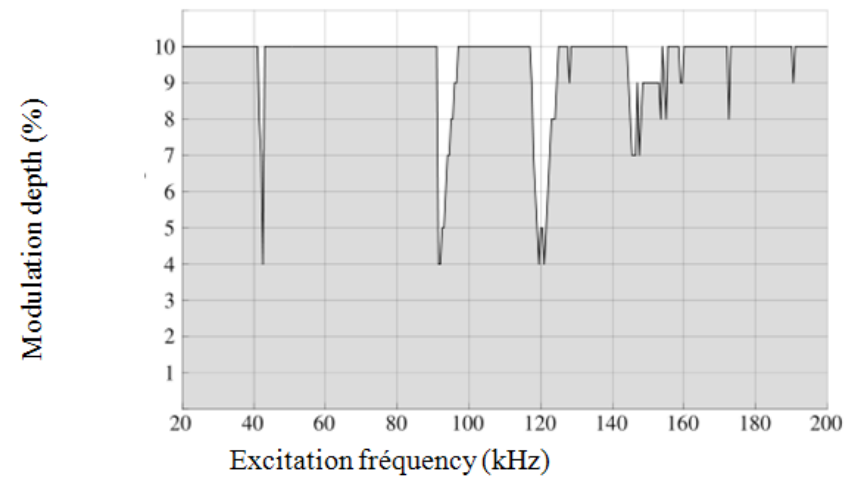

Figure II Experimental detection of acoustic instabilities over a wide frequency range.

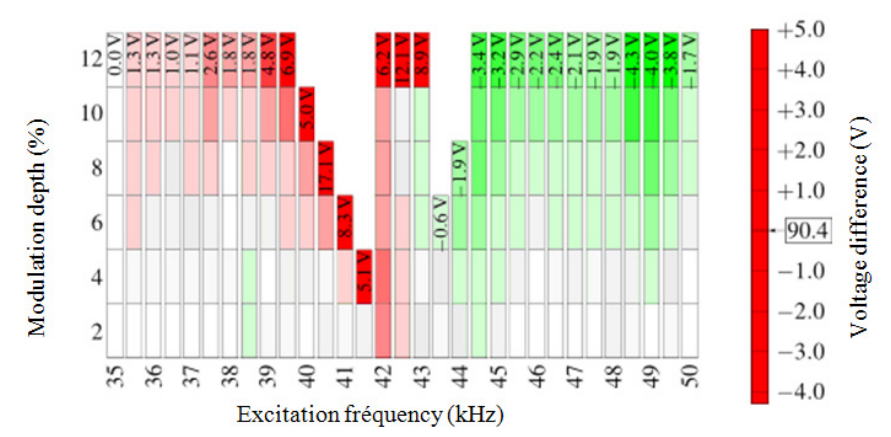

Figure 12 Measurement of the AR at the lowest excitation frequency at which arc flicker occurred.

For a more detailed analysis, the acoustic resonance at the lowest frequency, that shows arc flicker, was chosen in order to reduce the probability of measuring a superposition of acoustic eigenmodes. The frequency region of these detailed measurements ranges from $35 \mathrm{kHz}$ to $50 \mathrm{kHz}$ in $500 \mathrm{~Hz}$ steps, and the modulation depth was increased from $0 \%$ to $12 \%$ in $2 \%$ steps. Figure. 10 shows the detection of the acoustic resonance of a certain lamp. In addition to the threshold value of the modulation depth for each excitation frequency, the corresponding result of the measured voltage drop for each operating point is shown. In the scale, the reference voltage $\mathrm{V}_{\text {ref }}=90.4 \mathrm{~V}$ is pointed out. Measured voltages that are higher than $\mathrm{V}_{\text {ref }}$ are marked in red and operating points with a lower voltage are coloured green. The scale of the excess voltage is limited to $5.0 \mathrm{~V}$, which corresponds to $\mathrm{V}_{\text {lim }}$. Additionally, the measured voltage at the last operating point for each excitation frequency is presented. A higher voltage is the result of an increasing arc length so that the arc deflection increases as well. In contrast to that, the green colour indicates operating points with a decreasing arc deflection (arc straightening). The intensity of the colour represents the strength of the voltage difference to $\mathrm{V}_{\text {ref }}$ and, consequently, the strength of the deflection change. This was observed by eye and proved by measurements with the camera. In general, two different discharge arc behaviours can be observed in Figure 12 that is caused by different mechanisms. Up to $\mathrm{f}_{\mathrm{ex}}=42.5 \mathrm{kHz}$, the voltage increases when increasing the modulation depth. At higher excitation frequencies, the voltage decreases. The strength of the voltage difference to $\mathrm{V}_{\text {ref }}$ rises with increasing modulation depth because the share of the excitation part in relation to the stable part grows. The two local minima in the modulation depth are related to two different modes. The results of lamp highlight that the first minimum occurs at $f_{e x}=41.5 \mathrm{kHz}$ and the second minimum at $\mathrm{f}_{\text {ex }}=43.5 \mathrm{kHz}$. The voltage exceeds $\mathrm{V}_{\text {lim }}$ at $\mathrm{f}_{\text {ex }}=$ $41.5 \mathrm{kHz}$, whereas a fluctuating voltage $\mathrm{V}_{\text {fluc }}$ of a slightly straightened arc was detected at $f_{e x}=43.5 \mathrm{kHz}$. At the three measured frequencies between these two minima, the experiment terminated at $\alpha=12 \%$. The measured voltage at $\alpha=2 \%$ and $f_{\text {ex }}=42.0 \mathrm{kHz}$ is conspicuous because it is considerably higher than voltages at the same modulation depth at other excitation frequencies. The measurements serve to identify the mean values as well as the standard deviations of the reach able modulation depths and the frequencies, at which the lowest modulation depth is attained. Qualitatively, the results coincide with those presented before hand; especially the results shown in Figure $12 \mathrm{Up}$ to excitation frequencies, at which the lowest modulation depth occurs, the voltage increases with modulation depth. At higher excitation frequencies, arc straightening (decreasing voltage with 
increasing modulation depth), was detected. Quantitatively, the results differ from lamp to lamp of the same kind because geometry and gas composition tolerances occur in the manufacturing process.

\section{Conclusion}

The developed and verified simulation model facilitates the study of acoustic resonances that lead to light flicker in high intensity discharge lamps. The findings help to understand the underlying physical processes considerably better, which is crucial for an improvement of the lamp and driver design. The validated model enables development of new lamp systems that operate at stable conditions, possess an improved energy efficiency, are less bulky, are characterised by lower costs and have a reduced amount of mercury or even avoid this heavy metal. The simulation of instable discharge arc behaviour allows to design new electronic drivers that operate in the high frequency range and, therefore, have a significantly higher energy efficiency compared to state of the art lamps. Moreover, the model is helpful to identify acoustic eigenmodes that induce a fluid flow that causes arc straightening, which is equivalent to a stabilisation of the discharge arc and leads to a further increased energy efficiency. For further investigation of the acoustically induced streaming field in high intensity discharge lamps, some advancements are recommended. First, it would be beneficial to compare our results to additional experimental data. Especially, the velocity field inside the arc tube is of interest because it induces the arc flicker. Instead of an indirect detection of the fluid flow by voltage and light intensity measurements or by theoretical considerations, experimental results would be useful in benchmarking the simulation. The laser Doppler anemometry enables such measurements, but requires special lamp types with a transparent arc tube material and, therefore, necessitates a simulation model with a different geometry and adjusted transport coefficients. ${ }^{1-16}$

\section{Acknowledgements}

None.

\section{Conflicts of interest}

The authors declare that there is no conflict of interest.

\section{References}

1. Kettlitz M, Rarbach J, Zalach J. Investigation of acoustic resonances in high-power lamps. J Phys D: Appl Phys. 2011;44(14):145205.
2. Olsen J, Dreeben TD. Experimental and simulated straightening of metal halide arcs using power modulation. IEEE Trans Ind Appl. 2011;47(1):368-375.

3. Trestman GA. Minimizing cost of HID lamp electronic ballast. In Proc Ind Electron Soc Ann Conf. 2002:1214-1218.

4. National Electrical Manufacturers Association. NEMA standards publication LSD54-2010: The strengths and potentials of metal halide lighting systems. 2010.

5. Yan W, Ho EYK, Hui SYR. Investigation on methods of eliminating acoustic resonance in small wattage high-intensity discharge (HID) lamps. In Proc Ind Appl Soc Ann Meeting. 2000:3399-3406.

6. Stoffels WW, Baede AHFM, vander Mullen JAM, et al. Definition of a high intensity metal halide discharge reference lamp. Meas Sci Technol. 2006;17(11):N67-N70.

7. Stock wald K, Kaestle H, Weiss H. Significant efficacy enhancement of low wattage metal halide HID lamp systems by acoustically induced convection configuration. In Proc Int Conf Plasma Sci. 2008:1.

8. Stockwald K, Kaestle H, Ernst H. Highly efficient metal halide HID systems with acoustically stabilized convection. IEEE Trans Ind Appl. 2014;50(1):94-103.

9. U.S. Department of Energy. High intensity discharge lighting technology report. 2005.

10. Chhun L, Maussion P, Bhosle S, et al. Characterization of acoustic resonance in a high pressure sodium lamp. IEEE Trans Ind Appl. 2011;47(2):1071-1076.

11. Agilent Technologies, Inc. Agilent 33220A : 20 MHz Function/Arbitrary Wave form Generator User's Guide. 2007.

12. Wavetek Ltd. Maintenance Manual Model 29:10 MHz DDS Function Generator. 1997.

13. Yokogawa Electric Corporation. PZ4000 Power Analyzer User's Manual.

14. IDS Imaging Development Systems GmbH. USBUI 1120SE. 2015.

15. Tektronix,Inc. TDS1000 Band TDS2000B Series Digital Storage Oscilloscopes: User Manual. 2003.

16. Yan W, Hui SYR. Ageing effects on the stability performance of small wattage metal-halide (MH) lamps. In Proc Appl Power Electron Conf. 2004:978-983. 